\title{
Giant osmotic pressure in the forced wetting of hydrophobic nanopores
}

\author{
Millán Michelin-Jamois and Gérard Vigier \\ MATEIS, INSA-Lyon, CNRS UMR 5510, 69621 Villeurbanne, France \\ Cyril Picard ${ }^{\circledast}$ and Elisabeth Charlaix \\ Univ. Grenoble Alpes, LIPHY, F-38000 Grenoble, France
}

(Dated: June 10, 2021)

\begin{abstract}
The forced intrusion of water in hydrophobic nanoporous pulverulent material is of interest for quick storage of energy. With nanometric pores the energy storage capacity is controlled by interfacial phenomena. With subnanometric pores, we demonstrate that a breakdown occurs with the emergence of molecular exclusion as a leading contribution. This bulk exclusion effect leads to an osmotic contribution to the pressure that can reach levels never previously sustained. We illustrate on various electrolytes and different microporous materials, that a simple osmotic pressure law accounts quantitatively for the enhancement of the intrusion and extrusion pressures governing the forced wetting and spontaneous drying of the nanopores. Using electrolyte solutions, energy storage and power capacities can be widely enhanced.
\end{abstract}

Water in hydrophobic confinement is a topic of major importance for industrial applications, such as boiling, heat or mass transfers at interfaces [1-4], but also for the general understanding of hydrophobic interactions mediated by water in biological matter [5-7] and biomolecular responses under osmotic stress [8. Ordered hydrophobic nanoporous materials have been used to study water confined between hydrophobic surfaces in well defined geometries. But these material are also considered for novel energy applications. Due to the large specific area of nanoporous materials, of the order of $1000 \mathrm{~m}^{2} / \mathrm{cm}^{-3}$, and to the high tension of liquid/hydrophobic solid interfaces, forcing the intrusion of water in these materials provides a way to store energy in the form of interfacial free energy [3]. Then, the spontaneous extrusion of water out of the nanopores, related to their drying transition, allows the partial or full recovery of the stored energy.

In nanoporous materials of pore diameter larger than typically ten water molecules, it was shown that macroscopic concepts describe quantitatively the pressure $P_{\text {int }}$ at which the forced wetting, or intrusion, occurs. For instance in the cylindrical pores of hydrophobic mesoporous silicas, the intrusion pressure obeys the Laplace law of classical capillarity and scales as the inverse of the pore radius $1 / R_{p}[9]$. The drying transition on the other hand was shown to be triggered by the nucleation of nanobubbles, a mechanism also governed by interfacial phenomena [10 13. This nucleation process, in confinement, has also proven to be of interest to study the growth of nanobubbles, of precisely controlled shape, in relation with line tension effects.

With subnanometric pores, such as the pores of zeolithes or Metal Organic Frameworks, a breakdown occurs [14. In particular, electrolyte solutions exhibit huge wetting and drying pressures as compared to the ones of pure water. In this letter, we demonstrate for the first time that exclusion effects emerge as a leading contribution responsible for this giant pressure increase. We find that during the intrusion/extrusion processes, the pressure in the bulk electrolyte corresponds to the simple addition of the osmotic pressure $\Pi$ to the value of the intrusion/extrusion pressures obtained for pure water. This simple osmotic effect can increase very significantly the density of the stored energy.

Experiments have been carried out on a Metal Organic Framework (MOF) called Zeolitic Imidazolate Framework 8 (ZIF-8) 15, combined with seven different electrolyte solutions in various concentrations and temperature. ZIF-8 is commonly studied in the field of gas separation because of its high selectivity [16. An important feature of ZIF-8 is its natural hydrophobicity. Forced intrusion/extrusion cycles were achieved using a piston-cylinder device as described by Guillemot et al [17. (see figure 1), mounted on a traction/compression machine. Pressure-Volume (P-V) cycles were obtained on previously evacuated samples at constant temperature and under quasi-static conditions.

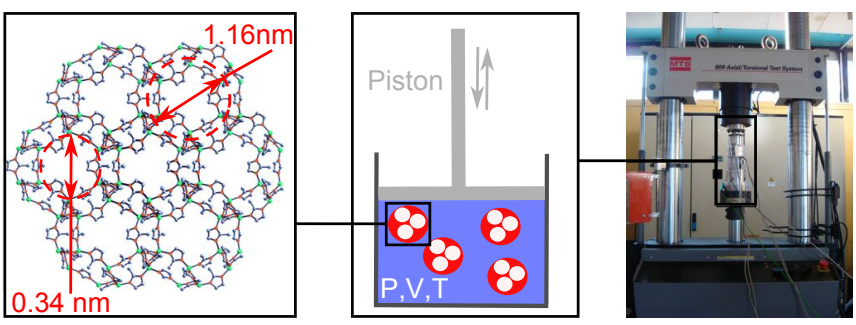

FIG. 1. Principle of forced intrusion/extrusion cycles. Pressure measurements are performed in an instrumented waterproof chamber, containing ZIF-8(red) [15] and electrolytes solution (blue), mounted on a traction machine.

The pressure of the liquid versus the intruded volume for a ZIF-8/water system is presented in the inset of figure 2. Intrusion and extrusion processes correspond to plateaus of the cycle. The value of the intrusion (resp. 
extrusion) pressure is defined as the average value on the intrusion (resp. extrusion) plateau. The ZIF-8/pure water system shows a small hysteresis with flat plateaus for both intrusion and extrusion. The intruded volume is about $500 \mathrm{~mm}^{3} \cdot \mathrm{g}^{-1}$. Values of the intrusion and ex-

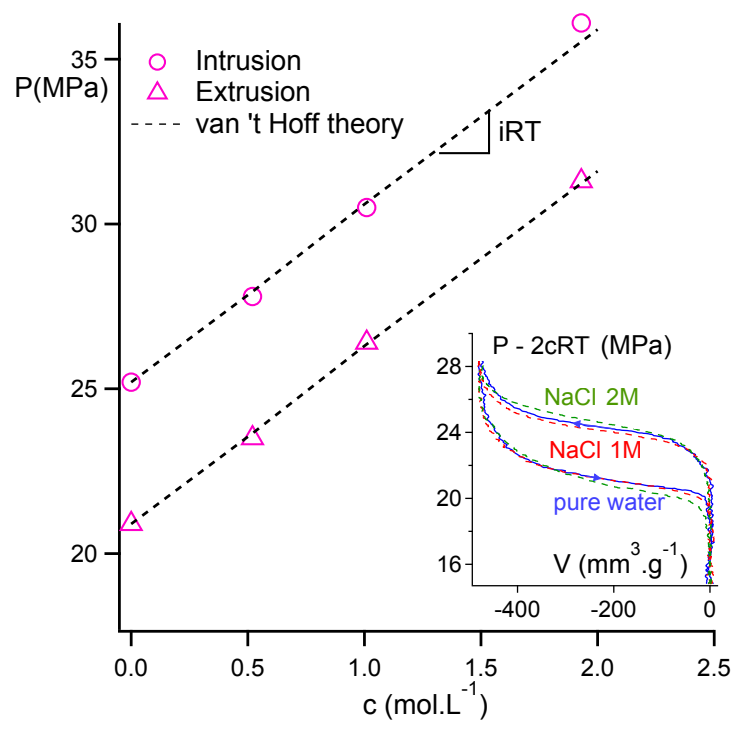

FIG. 2. Values of the intrusion and extrusion pressures in ZIF-8 as a function of the concentration $c$ of $\mathrm{NaCl}$ solutions at $323 \mathrm{~K}$. The inset shows the difference between the pressure and the van 't Hoff osmotic pressure versus intruded volume for $\mathrm{ZIF}-8 /$ water and two different $\mathrm{ZIF}-8 / \mathrm{NaCl}$ solutions at $T=343 \mathrm{~K}$.

trusion pressures are respectively $25.2 \pm 0.2 \mathrm{MPa}$ and $20.9 \pm 0.2 \mathrm{MPa}$ for this pure water system. The cycle obtained for $\mathrm{ZIF} 8 / \mathrm{NaCl}$ solutions reproduce perfectly the pure water cycle with a simple shift in pressure (see inset of figure 2). As shown in figure 2, the values of the intrusion pressure $P_{\text {int }}$ and the extrusion pressure $P_{\text {ext }}$ increase linearly with the $\mathrm{NaCl}$ concentration $c$, with slopes of respectively $5.5 \pm 0.2 \mathrm{MPa} \cdot \mathrm{M}^{-1}$ for intrusion and $5.2 \pm 0.2 \mathrm{MPa}^{\mathrm{M}} \mathrm{M}^{-1}$ for extrusion. This linear dependency corresponds very closely to the van 't Hoff law of osmotic pressure $\Pi$ :

$$
\Pi=i c R T
$$

where $R$ is the perfect gas constant, $T$ the temperature and $i$ the number of ions per electrolyte molecule. The corresponding slope is $i R T=5.37 \mathrm{MPa}^{-1}$ for $i=2$ and $T=323 K$. This theoretical value is very close to the slopes of the experimental data.

This behaviour unravels the mechanism at work in the intrusion-extrusion process of the $\mathrm{NaCl}$ solutions. The ZIF-8 behaves as an almost ideal sieve for $\mathrm{Na}^{+}$and $\mathrm{Cl}^{-}$ ions, as predicted by molecular simulations 18 . Because of this selectivity, only water penetrates into the material, as sketched in figure 3. The pressure difference between the pure water inside the material and the surrounding solution is the osmotic pressure. This pressure difference is constant through the whole process, therefore the electrolyte cycles are shifted replicas of the pure water cycles, as only pure water is present into the material. In other words, the pressure shift observed with the electrolyte solution is the additional pressure required to extract pure water from the surrounding electrolyte.

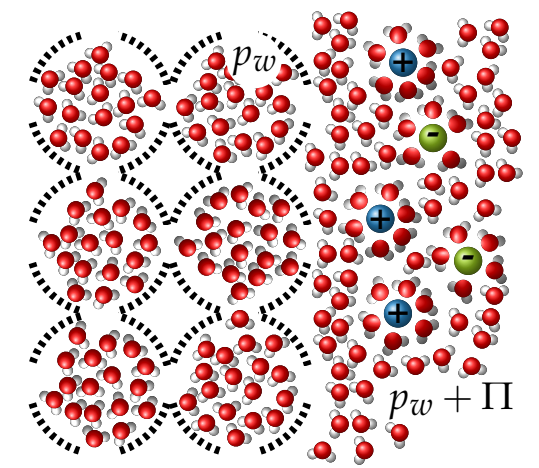

FIG. 3. Schematic view of the ion exclusion from the ZIF8 microporous material leading to an osmotic contribution. The jump between the pressure $p_{w}$ inside the pores of a microporous particle and the pressure $p_{w}+\Pi$ outside the pores is the osmotic pressure $\Pi$.

We expect the same scenario to hold for all cases in which ions are perfectly excluded from the microporous material. We have studied seven electrolytes: lithium chloride $\mathrm{LiCl}$, lithium iodide LiI, sodium chloride $\mathrm{NaCl}$, sodium bromide $\mathrm{NaBr}$, sodium iodide $\mathrm{NaI}$, calcium chloride $\mathrm{CaCl}_{2}$ and cesium chloride $\mathrm{CsCl}$. For each solution, the excess pressure with respect to pure water, $\Delta P=P^{\text {solution }}-P^{\text {water }}$ was determined both for intrusion and for extrusion at different temperatures [19]. Figure 4 summarizes the results with the experimental $\Delta P$ 's plotted versus the van 't Hoff pressure $i c R T$. The figure also includes data obtained in Silicalite-1 zeolite with $\mathrm{NaCl}$ and $\mathrm{LiCl} 14$ and data obtained in $\mathrm{ZIF}-8$ with $\mathrm{NaCl}, \mathrm{LiCl}$ and $\mathrm{KCl}[20$. The case of NaI and LiI is discussed later and reported in figure 5. As predicted by van't Hoff law, data points obtained for low concentrations of $\mathrm{LiCl}, \mathrm{NaCl}, \mathrm{NaBr}$ and $\mathrm{CsCl}$, align along a straight line whose slope is 1 .

Measurable deviations from the van 't Hoff law are observed with saturating solutions of $\mathrm{NaCl}$ and $\mathrm{LiCl}$ in Silicalite-1 zeolites (Tzanis data) as well as with $\mathrm{CaCl}_{2}$ $0.5 \mathrm{M}$ in ZIF-8 (our data). The deviating pressures are higher than predicted by the van 't Hoff law. We attribute these deviations to non-ideal effects in the electrolyte solutions. Non-ideal effects are expected when the Coulomb interaction energy between ions becomes comparable to the thermal energy. This occurs for a typical distance between ions scaling as $z^{+} z^{-} \lambda_{B}$ with $z^{+}$and $z^{-}$ 


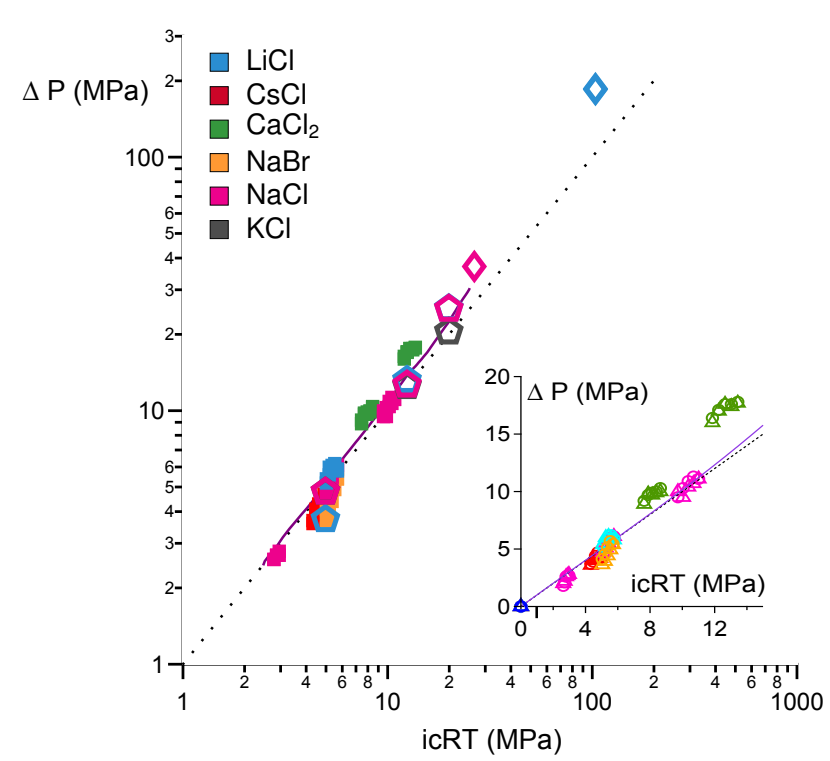

FIG. 4. Excess intrusion and extrusion pressures with respect to pure water measured for the electrolytes mentioned in the legend. The $\mathrm{x}$-scale is the van 't Hoff osmotic pressure $\Pi=i c R T$ with $i$ the number of ions per salt molecule and $c$ the salt concentration. The values of our own data ( $\mathbf{a})$ are listed in supplementary Tables 1 to 5 [19. $(\diamond)$ are data from Tzanis et al [14, $(\triangle)$ are data from Ortiz et al [20]. The straight line $(\cdots)$ corresponds to the van 't Hoff law of osmotic pressure. The continuous line $(-)$ is a simulation of the osmotic pressure of $\mathrm{NaCl}$ solutions from Luo et al [21]. The inset is a magnification of our data with $(\bigcirc)$ intrusion pressure and $(\triangle)$ extrusion pressure.

the ions valence and $\lambda_{B}$ the Bjerrum length:

$$
\lambda_{B} \sim \frac{e^{2}}{4 \pi \epsilon_{0} \epsilon_{r} k_{B} T}
$$

with $\epsilon_{0}$ the vacuum permitivity, $\epsilon_{r}$ the water dielectric constant and $k_{B}$ the Boltzmann constant. The distance $z^{+} z^{-} \lambda_{B}$ is related to a limit salt concentration $c_{z^{+} z^{-}}$:

$$
c_{z^{+} z^{-}}=\frac{1}{i \mathcal{N}_{A}} \frac{1}{\left(z^{+} z^{-} \lambda_{B}\right)^{3}}=\frac{1}{i \mathcal{N}_{A}}\left(\frac{4 \pi \epsilon_{0} \epsilon_{r} k_{B} T}{z^{+} z^{-} e^{2}}\right)^{3}
$$

and a limit pressure $\Pi_{z^{+} z^{-}}=i c_{z^{+} z^{-}} R T$ above which correlation effects between ions can not be neglected. For monovalent ions at the lowest considered temperature $T=303 \mathrm{~K}$, the limit concentration is around $c_{11}=1 \mathrm{M}$, corresponding to a limit pressure $\Pi_{11}$ of about $5.5 \mathrm{MPa}$. At concentrations much higher than $c_{11}$ the osmotic pressure is expected to increase more rapidly than the linear van't Hoff prediction. This is the range of concentration above which deviations are experimentally observed for $\mathrm{NaCl}$ and $\mathrm{LiCl}$ in figure 4. The molecular dynamic simulation of the osmotic pressure of $\mathrm{NaCl}$ carried out by Luo et al 21] indeed meets the data point measured by
Tzanis et al and Ortiz et al for saturating $\mathrm{NaCl}$ solutions in zeolithes [14, 20]. Taking into account the trend of the simulation, it is safe to propose that osmotic pressure is also the good candidate to explain the much higher pressure measured by the same authors for concentrated $\mathrm{LiCl}$ solutions (180 MPa of excess pressure at saturation).

The valence of the ions has a major impact on $\Pi_{z^{+} z^{-}}$. For a salt with one divalent ion and one monovalent ion such as $\mathrm{CaCl}_{2}$, the limit concentration is $c_{21}=0.15 \mathrm{M}$, and the corresponding pressure is $\Pi_{21}=1.2 \mathrm{MPa}$. Accordingly, the experimental values of $\Delta P$ measured for $\mathrm{CaCl}_{2}$ solutions are larger than the van't Hoff theoretical curve in figure 4, and the data points go away from the van't Hoff law as the concentration increases, in a similar way to what is observed with monovalent ions at higher concentration.

Remains the particular case of $\mathrm{NaI}$ and LiI solutions depicted on figure 5. The behaviour of these salts is very different from the previous ones. The cycle of the NaI $0.94 \mathrm{M}$ solution is compared to one of pure water in the inset of figure 5 . The electrolyte cycle is not anymore a simple translation of the pure water cycle. The excess of the intrusion pressure is much larger than the excess of the extrusion pressure, and the cycle hysteresis is larger for the electrolyte solution. A similar result is obtained with all concentrations of $\mathrm{NaI}$ and LiI solutions as shown in figure 5. Furthermore the excess pressures measured in the electrolyte cycle with respect to pure water are below the van 't Hoff osmotic pressure.

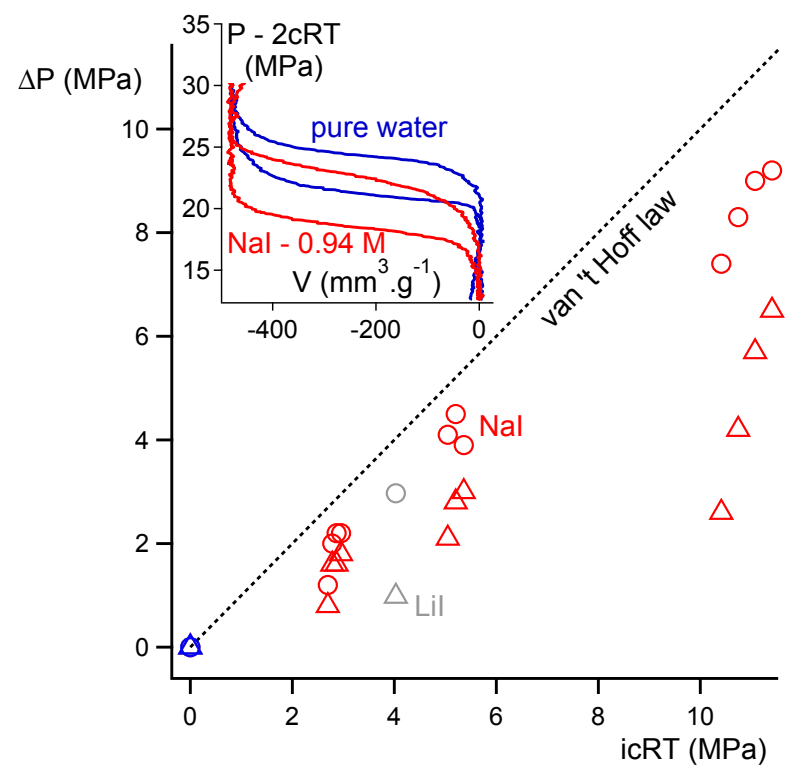

FIG. 5. Excess intrusion pressure $(\bigcirc)$ and excess extrusion pressure $(\triangle)$ of $\mathrm{NaI}$ and $\mathrm{LiI}$ solutions in ZIF-8 with respect to pure water, plotted as a function of the van 't Hoff osmotic pressure $i c R T$. Inset : difference between the pressure and the van 't Hoff osmotic pressure of ZIF-8/water (blue) and ZIF-8/NaI - 0.94M (red) at $343 \mathrm{~K}$. 
We attribute this behavior to the partial penetration of ions inside the micropores. As a result the interfacial energies are expected to be different from the one of pure water, and consequently the shape of the cycle is also different. Furthermore the pressure difference between the surrounding electrolyte and the intruding liquid is less than the van 't Hoff osmotic pressure as the intruding liquid is not pure water. The penetration of these ions into the pores is far from intuitive as the radius of the hydrated $\mathrm{I}^{-}, r_{H}=3.3 \AA 22$, is twice larger than the radius of the smallest ZIF-8 pores. The partial penetration of $\mathrm{I}^{-}$ions in ZIF-8 is certainly due to their large polarisability, responsible for their deformability and affinity for hydrophobic surfaces 23, 24.

In any case, the transition between ion intrusion and exclusion is not related to electrostatic screening. Iodide ions enter the pores for small concentrations corresponding to Debye length larger than the pore size while other ions are still excluded from the pore at high concentrations corresponding to Debye length smaller than $0.1 \mathrm{~nm}$.

In summary, our results show that electrolyte solutions can exhibit giant intrusion/extrusion pressures in micropores due to osmotic effects. As ions are repelled from the pores rather than confined in them, the intrusion process is a three-dimensional extraction of pure water from the electrolyte solution. This osmotic mechanism allows to increase the pressure up to one order of magnitude compared to the intrusion/extrusion pressure obtained with pure water. The intrusion process is associated to a storage of mechanical energy, equal to the product of the macroscopic pressure times the intruded volume 25. As a result the energy stored in the pores per unit volume can be higher than with ultracapacitors. Morevover, it was demonstrated that the intrusion/extrusion pressures depend weakly on the rate of the process due the very short time scale of wetting and drying transitions [12]. Therefore our results pave the way towards the design of lyophobic energy storage systems with targeted powerdensities as large as $50 \mathrm{~kW}$ per solution liter that is one order of magnitude larger than with ultracapacitors.

From a more fundamental point of view, our results demonstrate experimentally that the concept of osmotic pressure can be extended up to giant values of hundreds of MPa that have never been reached with conventional separation membranes. The selective pulverulent nanomaterial act as a volumic membrane able to sustain huge osmotic pressures because each individual material particle is submitted to a uniform osmotic pressure. Finally, the forced intrusion of aqueous solution in hydrophobic nanoporous material can be put forward as a way to probe mechanically molecular interactions. This mechanical characterization offers a new way to probe the stability of the hydration layer of ions and the affinity of ions for hydrophobic surfaces as exemplified with the difference of behaviors between iodide ions and other anions.
The authors kindly thank Lydéric Bocquet and Benoit Coasne for fruitful discussions. We thanks also Airbus Defence and Space, the French Direction Générale de l'Armement, the AGIR funding from the University Grenoble Alpes and the French National Agency for Research (ANR-14-CE05-0017) which support our researches.

cyril.picard@ujf-grenoble.fr

[1] R. Chen, M.-C. Lu, V. Srinivasan, Z. Wang, H. H. Cho, and A. Majumdar, Nano letters 9, 548 (2009).

[2] X. Zhang, H. Lhuissier, C. Sun, and D. Lohse, Physical Review Letters 112, 144503 (2014).

[3] V. Eroshenko, R. Regis, M. Soulard, and J. Patarin, J. Am. Chem. Soc. 123, 8129 (2001)

[4] C. Suciu, T. Iwatsubo, and S. Deki, J. Colloid Interface Sci. 259, 62 (2003).

[5] B. J. Berne, J. D. Weeks, and R. Zhou, Annual Review of Physical Chemistry 60, 85 (2009).

[6] P. R. ten Wolde and D. Chandler, Proceedings of the National Academy of Sciences of the United States of America 99, 6539 (2002).

[7] Y. Zhou, W. Guo, and L. Jiang, Physics, Mechanics and Astronomy 57, 836 (2014).

[8] P. Masson, S. Lushchekina, L. M. Schopfer, and O. Lockridge, Biochem. J. 454, 387 (2013).

[9] B. Lefevre, A. Saugey, J. Barrat, L. Bocquet, E. Charlaix, P. Gobin, and G. Vigier, Colloids Surfaces A Physicochem. Eng. Asp. 241, 265 (2004).

[10] K. Leung, A. Luzar, and D. Bratko, Physical Review Letters 90, 065502 (2003)

[11] S. Sharma and P. G. Debenedetti, Proceedings of the National Academy of Sciences of the United States of America 109, 4365 (2012).

[12] L. Guillemot, T. Biben, A. Galarneau, G. Vigier, and E. Charlaix, Proceedings of the National Academy of Sciences 109 (2012), 10.1073/pnas.1207658109

[13] Y. Grosu, O. Ievtushenko, V. Eroshenko, J. Nedelec, and J. Grolier, Colloids Surf. A 441, 549 (2014)

[14] L. Tzanis, H. Nouali, T. Daou, M. Soulard, and J. Patarin, Mater. Lett. 115, 229 (2014)

[15] K. S. Park, Z. Ni, A. P. Côté, J. Y. Choi, R. Huang, F. J. Uribe-romo, H. K. Chae, M. O'Keeffe, and O. M. Yaghi, Proc. Natl. Acad. Sci. 103, 8 (2006).

[16] S. Yang, X. Lin, W. Lewis, M. Suyetin, E. Bichoutskaia, J. E. Parker, C. C. Tang, D. R. Allan, P. J. Rizkallah, P. Hubberstey, N. R. Champness, K. M. Thomas, A. J. Blake, and M. Schröder, Nat. Mater. 11, 710 (2012).

[17] L. Guillemot, A. Galarneau, G. Vigier, T. Abensur, and E. Charlaix, Rev. Sci. Instrum. 83, 105105 (2012).

[18] Z. Hu, Y. Chen, and J. Jiang, J. Chem. Phys. 134 (2011), 10.1063/1.3573902

[19] See Supplemental Material which includes Refs. $[12,15,26]$.

[20] G. Ortiz, H. Nouali, C. Marichal, G. Chaplais, and J. Patarin, J. Phys. Chem. C 118, 7321 (2014).

[21] Y. Luo and B. Roux, J. Phys. Chem. Lett. 1, 183 (2010)

$[22]$ B. E. Conway, Ionic hydration in chemistry and biophysics, 12th ed. (Elsevier, 1981). 
[23] D. Huang, C. Cottin-Bizonne, C. Ybert, and L. Bocquet, Phys. Rev. Lett. 98 (2007), 10.1103/PhysRevLett.112.068103

[24] P. Jungwirth and D. J. Tobias, Chem. Rev. 106, 1259
(2006).

[25] V. Eroshenko and Y. F. Lazarev, J. Appl. Mech. Tech. Phys. 53, 98 (2012).

[26] E. P. Brunauer, S. and E. Teller, J. Am. Chem. Soc. 60, 309 (1938). 